\title{
Prevalence of Antimicrobial Resistance among 723 Outpatient Clinical Isolates of Moraxella catarrhalis in the United States in 1994 and 1995: Results of a 30-Center National Surveillance Study
}

\author{
GARY V. DOERN, ${ }^{*}$ ANGELA B. BRUEGGEMANN,${ }^{1}$ GARY PIERCE, ${ }^{1}$ TRICIA HOGAN, ${ }^{1}$ \\ H. PRESTON HOLLEY, JR., ${ }^{2}$ AND ALAN RAUCH ${ }^{2}$ \\ Clinical Microbiology Laboratories, University of Massachusetts, Worcester, Massachusetts $01655,{ }^{1}$ and \\ Medical Affairs, Glaxo Wellcome, Inc., Research Triangle Park, North Carolina $27709^{2}$
}

Received 28 May 1996/Returned for modification 27 August 1996/Accepted 6 October 1996

\begin{abstract}
Seven hundred twenty-three isolates of Moraxella catarrhalis obtained from outpatients with a variety of infections in 30 medical centers in the United States between 1 November 1994 and 30 April 1995 were characterized in a central laboratory. The overall rate of $\beta$-lactamase production was $95.3 \%$. When the National Committee for Clinical Laboratory Standards MIC interpretive breakpoints for Haemophilus influenzae were applied, percentages of strains found to be susceptible to selected oral antimicrobial agents were as follows: azithromycin, clarithromycin, and erythromycin, 100\%; tetracycline and chloramphenicol, 100\%; amoxicillin-clavulanate, 100\%; cefixime, 99.3\%; cefpodoxime, 99.0\%; cefaclor, $99.4 \%$; loracarbef, 99.0\%; cefuroxime, $98.5 \%$; cefprozil, $94.3 \%$; and trimethoprim-sulfamethoxazole, $93.5 \%$.
\end{abstract}

Moraxella catarrhalis is now recognized as a common cause of a variety of localized, community-acquired infections, in particular, acute otitis media, maxillary sinusitis, and acute purulent exacerbation of chronic bronchitis $(3,7,10)$. Most clinical isolates of $M$. catarrhalis are found to produce one of two $\beta$-lactamases, BRO-1 and BRO-2 $(5,8,11,12)$. Both of these enzymes hydrolyze penicillin, ampicillin, and amoxicillin, although to differing degrees (i.e., BRO-1 hydrolyzes them to a greater extent than BRO-2) $(5,11)$. As a result, MICs of penicillin, ampicillin, and amoxicillin are elevated for $\beta$-lactamase-producing strains of $M$. catarrhalis, especially BRO-1 enzyme producers $(1,4,6,12)$. Whether production of either enzyme is associated with clinical failures in patients treated with these $\beta$-lactams has not been determined. Until such information is available, however, prudence would dictate that all infections caused by $\beta$-lactamase-producing $M$. catarrhalis, irrespective of which enzyme is produced, be considered refractile to management with penicillin, ampicillin, or amoxicillin.

Two recent large multicenter surveillance studies in the United States revealed overall rates of $\beta$-lactamase production of $84.1 \%$ in 1987 and 1988 (6) and $92.0 \%$ in 1992 and 1993 (1). Interestingly, there is some evidence that prior to 1976, in both the United States and Europe, M. catarrhalis rarely if ever produced $\beta$-lactamase (12).

Because the $\beta$-lactamases of $M$. catarrhalis are inhibited by clavulanate, the combination drug amoxicillin-clavulanate has been consistently active against this species $(1,4,6)$. The same is true of oral cephalosporins, excepting cephalexin and cefadroxil (4). Erythromycin and tetracycline resistance has been reported (2); however, the two most recent countrywide surveillance studies in the United States during 1987 and 1993 failed to identify a single macrolide- or tetracycline-resistant

\footnotetext{
* Corresponding author. Mailing address: Clinical Microbiology Laboratories, University of Massachusetts Medical Center, 55 Lake Ave. North, Worcester, MA 01655. Phone: (508) 856-6417. Fax: (508) 856-1537.
}

strain among a total of $>1,000$ isolates of $M$. catarrhalis. In contrast, trimethoprim-sulfamethoxazole (TMP-SMX) resistance is being reported with greater frequency (1).

The intent of the present study was to systematically determine the prevalence of antimicrobial resistance among current isolates of $M$. catarrhalis in the United States. Between 1 November 1994 and 30 April 1995, a total of 723 different isolates of this organism were prospectively collected from various specimens from outpatients in 30 different U.S. medical centers. For further characterization, isolates were transported to the University of Massachusetts Medical Center on rayon swabs immersed in $12 \mathrm{ml}$ of Amies semisolid transport medium containing charcoal. Stock cultures were prepared with an absorbent-bead system (ProLab Diagnostics, Austin, Tex.), and organisms were stored at $-70^{\circ} \mathrm{C}$ until further use. All organisms were subcultured twice on sheep blood agar plates prior to further characterization. Isolates were confirmed as $\mathrm{M}$. $\mathrm{ca}$ tarrhalis on the basis of Gram stain morphology and production of oxidase and butyric acid esterase.

Susceptibility studies. MICs were determined by a broth microdilution procedure $(100-\mu l$ total volume per well; final inoculum concentration, ca. $\left.5 \times 10^{5} \mathrm{CFU} / \mathrm{ml}\right)$ in cation-adjusted Mueller-Hinton broth (Difco Laboratories, Detroit, Mich.), with trays incubated at $35^{\circ} \mathrm{C}$ in ambient air for 22 to $24 \mathrm{~h}$ prior to determination of results. Sixteen antibiotics, obtained from their respective manufacturers as laboratory-grade powders, were each tested in 12 different concentrations in an attempt to limit the number of off-scale results. The antimicrobial agents were penicillin, ampicillin, amoxicillin, amoxicillin-clavulanate (2:1), cefaclor, loracarbef, cefprozil, cefuroxime, cefixime, cefpodoxime, erythromycin, azithromycin, clarithromycin, TMPSMX (1:19), chloramphenicol, and tetracycline. Staphylococcus aureus ATCC 29213 and Escherichia coli ATCC 25922 were used as controls. $\beta$-Lactamase production was assessed with all isolates by the nitrocefin disk assay (Cefinase; Becton Dickinson Microbiology Systems, Cockeysville, Md.).

A total of 723 isolates of $M$. catarrhalis were characterized (mean number contributed per medical center $=24.1$; range $=$ 
TABLE 1. MICs of 16 antimicrobial agents obtained with 723 recent clinical isolates of $M$. catarrhalis

\begin{tabular}{|c|c|c|c|c|c|c|}
\hline \multirow{2}{*}{ Antimicrobial agent } & \multicolumn{3}{|c|}{$\beta$-Lactamase-positive isolates $(n=689)$} & \multicolumn{3}{|c|}{$\beta$-Lactamase-negative isolates $(n=34)$} \\
\hline & $\mathrm{MIC}_{50}{ }^{a}$ & $\mathrm{MIC}_{90}{ }^{a}$ & MIC range & $\mathrm{MIC}_{50}$ & $\mathrm{MIC}_{90}$ & MIC range \\
\hline Penicillin & 16 & 32 & $0.25-\geq 32$ & 0.03 & 0.03 & $\leq 0.015-0.25$ \\
\hline Ampicillin & 4 & 16 & $0.06->32$ & $\leq 0.015$ & 0.03 & $\leq 0.015-0.06$ \\
\hline Amoxicillin & 4 & 16 & $0.06->32$ & $\leq 0.015$ & 0.06 & $\leq 0.015-0.12$ \\
\hline Amoxicillin-clavulanate & 0.25 & 0.5 & $\leq 0.015-2$ & $\leq 0.015$ & 0.06 & $\leq 0.015-0.12$ \\
\hline Cefaclor & 1 & 2 & $0.03-16$ & 0.5 & 0.5 & $0.12-0.5$ \\
\hline Loracarbef & 1 & 4 & $0.06-64$ & 0.25 & 0.25 & $0.12-0.5$ \\
\hline Cefprozil & 4 & 8 & $0.06-64$ & 1 & 1 & $0.25-1$ \\
\hline Cefuroxime & 2 & 4 & $0.06-32$ & 0.5 & 0.5 & $0.12-1$ \\
\hline Cefixime & 0.25 & 0.5 & $\leq 0.015-4$ & 0.03 & 0.06 & $\leq 0.015-0.25$ \\
\hline Cefpodoxime & 1 & 2 & $0.06-8$ & 0.12 & 0.25 & $0.06-1$ \\
\hline Erythromycin & 0.25 & 0.25 & $0.06-1$ & 0.12 & 0.25 & $0.06-0.25$ \\
\hline Azithromycin & 0.06 & 0.06 & $0.03-0.12$ & 0.06 & 0.06 & $0.03-0.12$ \\
\hline Clarithromycin & 0.06 & 0.12 & $0.03-0.5$ & 0.06 & 0.12 & $0.03-0.12$ \\
\hline TMP-SMX & 0.5 & 0.5 & $\leq 0.06-8$ & 0.25 & 0.5 & $\leq 0.06-0.5$ \\
\hline Chloramphenicol & 0.5 & 1 & $0.25-2$ & 0.5 & 1 & $0.5-1$ \\
\hline Tetracycline & 0.5 & 0.5 & $\leq 0.06-1$ & 0.5 & 0.5 & $\leq 0.06-0.5$ \\
\hline
\end{tabular}

${ }^{a} \mathrm{MIC}_{50}$ and $\mathrm{MIC}_{90}$, MICs at which 50 and $90 \%$ of isolates, respectively, are inhibited.

14 to 43 ). Males were the source of $57.2 \%$ of isolates. The percentages of isolates obtained from patients in different age groups were as follows: 0 to 5 years, $33.3 \%$; 6 to 10 years, $4.8 \%$; 11 to 20 years, $3.1 \%$; 21 to 50 years, $18.2 \%$; and $>50$ years, $40.6 \%$. The percentages of isolates obtained from different specimens were as follows: middle ear fluid, $4.5 \%$; sinus aspirates, $5.4 \%$; conjunctival specimens, $7.4 \%$; lower respiratory tract, $80.3 \%$; blood, $1.7 \%$; and other, $0.8 \%$.

Of the 723 isolates, $689(95.3 \%)$ produced $\beta$-lactamase. MIC results for 16 antimicrobial agents are listed in Table 1 . MICs of all $10 \beta$-lactam antimicrobial agents examined in this study were higher for $\beta$-lactamase-positive than $\beta$-lactamase-negative strains. Overall, ampicillin was equivalent to amoxicillin in activity; both were more active than penicillin. The National Committee for Clinical Laboratory Standards (NCCLS) currently does not provide MIC interpretive breakpoints for $M$. catarrhalis (9). If current NCCLS MIC breakpoints for Haemophilus influenzae (9) were applied, then $100 \%$ of the test strains would have been categorized as susceptible to amoxicillin-clavulanate.

As can be seen from the range of MICs listed in Table 1, not all $\beta$-lactamase-positive strains would have been categorized as ampicillin resistant according to the NCCLS Haemophilus breakpoint of $\geq 4 \mu \mathrm{g} / \mathrm{ml}$ (9). Indeed, for $136(19.7 \%)$ of the 689 $\beta$-lactamase-positive isolates, the MICs were $\leq 2 \mu \mathrm{g} / \mathrm{ml}$. It is likely that these strains produced the BRO-2 enzyme, which is known to be associated with low penicillin and ampicillin MICs due to small amounts of enzyme produced and low substrate affinity $(5,11)$

The activities of six oral cephalosporins were also examined in this study. On the basis of current NCCLS Haemophilus breakpoints (9), the percentages of strains determined to be susceptible to the cephalosporins were $99.3 \%$ for cefixime, $99.0 \%$ for cefpodoxime, $99.4 \%$ for cefaclor, $99.0 \%$ for loracarbef, $98.5 \%$ for cefuroxime, and $94.3 \%$ for cefprozil. All nonsusceptible strains produced $\beta$-lactamase. With the exception of the cefprozil MICs, the cephalosporin MICs obtained with those strains categorized as not susceptible were only a single doubling concentration higher than the susceptible MIC breakpoints for the respective antimicrobial agents. In the case of cefprozil, for 12 isolates ( $1.6 \%$ of the total) the MICs were $\geq 32$ $\mu \mathrm{g} / \mathrm{ml}$, and these isolates would have been categorized as re- sistant according to current NCCLS breakpoints for $H$. influenzae (9).

Again on the basis of current Haemophilus breakpoints (9), all isolates of $M$. catarrhalis were susceptible to the macrolides examined in this study (erythromycin, azithromycin, and clarithromycin). The same was true of chloramphenicol and tetracycline. With TMP-SMX, the MICs for $6.5 \%$ of the isolates were $\geq 1.0 \mu \mathrm{g} / \mathrm{ml}$, and therefore, those isolates would have been classified as intermediate (MICs 1 to $2 \mu \mathrm{g} / \mathrm{ml} ; n=41$ ) or resistant (MICs, $\geq 4 \mu \mathrm{g} / \mathrm{ml} ; n=6$ ) according to the NCCLS Haemophilus interpretive breakpoints (9).

It is apparent from the results of this study that today nearly all clinical isolates of $M$. catarrhalis produce $\beta$-lactamase and as a result should probably be managed with agents other than penicillin, ampicillin, or amoxicillin. Fortunately, this organism remains uniformly susceptible to amoxicillin-clavulanate and nearly uniformly susceptible to five oral cephalosporins, cefixime, cefpodoxime, cefaclor, loracarbef, and cefuroxime. Among these five agents, cefixime is clearly the most active.

Among non- $\beta$-lactam agents, three macrolides (azithromycin, clarithromycin, and erythromycin), tetracycline, and chloramphenicol were uniformly active; all strains were susceptible. That was not true of TMP-SMX, to which only $93.5 \%$ of strains were susceptible. Taken collectively, the results of this surveillance study suggest that although rates of $\beta$-lactamase production have increased slightly, $M$. catarrhalis has not changed substantially in the context of antimicrobial resistance during the period since the last large U. S. multicenter surveillance study in 1992 and 1993 (3). A variety of oral antimicrobial agents remain suitable for the management of outpatient $M$. catarrhalis infections.

We thank Brendan Curley for technical assistance, Greg Giguere for statistical analysis, and Debbie McQuaid for excellent secretarial support. In addition, we thank the following individuals for provision of clinical isolates of $M$. catarrhalis: Melodie Beard, Rush-Presbyterian St. Luke's Medical Center; Paul Bourbeau, Geisinger Medical Center; Joseph Campos, Children's Hospital National Medical Center; Kimberle Chapin, University of South Alabama; Carla Clausen, Seattle Children's Hospital; Frank Cockerill, Mayo Clinic; Judy Daly, Primary Children's Medical Center; Gerald Dennis, Methodist Hospital; Phyllis Della-Latta, Columbia Presbyterian Hospital; Michael Dunne, Henry Ford Hospital; Peter Gilligan, University of North Carolina 
School of Medicine; Paul Granato, SUNY Health Sciences CenterSyracuse; Dwight Hardy, Strong Memorial Hospital; Bob Jerris, Dekalb General Hospital; Karen Sue Kehl, Milwaukee Children's Hospital; Susan Marrone, Kaiser Hospital-Portland; Pat Mickelson, Stanford University Medical Center; Margie Morgan, Cedars-Sinai Hospital; Ann Robinson, Hartford Hospital; Susan Rossmann, Texas Children's Hospital, Dan Sahm, Jewish Hospital; Michael Saubolle, Good Samaritan Hospital; Susan Sharp, Mt. Sinai Hospital; Paul Southern, University of Texas Southwestern Medical Center; Ronald St. Amand, University of Massachusetts Medical Center; Richard Thompson, Evanston Hospital; Grace Thorne, Boston Children's Hospital; Allan Truant, Temple University School of Medicine; John Washington, Cleveland Clinic; and Michael Wilson, Denver General Hospital.

This study was supported by a grant from Glaxo Wellcome, Inc.

\section{REFERENCES}

1. Barry, A. L., M. A. Pfaller, P. C. Fuchs, and R. R. Packer. 1994. In vitro activities of 12 orally administered antimicrobial agents against four species of bacterial respiratory pathogens from U. S. medical centers in 1992 and 1993. Antimicrob. Agents Chemother. 38:2419-2425.

2. Brown, B. A., R. J. Wallace, Jr., C. W. Flanagan, R. W. Wilson, J. I. Luman, and S. D. Redditt. 1989. Tetracycline and erythromycin resistance among clinical isolates of Branhamella catarrhalis. Antimicrob. Agents Chemother. 33:1631-1633.

3. Doern, G. V. 1986. Branhamella catarrhalis-an emerging human pathogen. Diagn. Microbiol. Infect. Dis. 4:191-201.
4. Doern, G. V., and T. A. Tubert. 1988. In vitro activities of 39 antimicrobial agents for Branhamella catarrhalis and comparison of results with different quantitative susceptibility test methods. Antimicrob. Agents Chemother. 32: 259-261.

5. Farmer, T., and C. Reading. 1982. $\beta$-Lactamases of Branhamella catarrhali and their inhibition by clavulanic acid. Antimicrob. Agents Chemother. 21: 506-508.

6. Jorgensen, J. H., G. V. Doern, L. A. Maher, A. W. Howell, and J. S. Redding 1990. Antimicrobial resistance among respiratory isolates of Haemophilus influenzae, Moraxella catarrhalis, and Streptococcus pneumoniae in the United States. Antimicrob. Agents Chemother. 34:2075-2080.

7. Marchant, C. D. 1990. Spectrum of disease due to Branhamella catarrhalis in children with particular reference to acute otitis media. Am. J. Med. 88(5A): 15S-19S.

8. Nash, D. R., R. J. Wallace, Jr., V. A. Steingrube, and P. A. Shurin. 1986. Isoelectric focusing of $\beta$-lactamases from sputum and middle ear isolates of Branhamella catarrhalis recovered in the United States. Drugs 31:48-54.

9. National Committee for Clinical Laboratory Standards. 1995. Methods for dilution antimicrobial susceptibility tests for bacteria that grow aerobically, 4th ed. M7-A4. National Committee for Clinical Laboratory Standards, Villanova, Pa.

10. Sarubbi, F. A., J. W. Myers, J. J. Williams, and C. G. Shell. 1990. Respiratory infections caused by Branhamella catarrhalis. Am. J. Med. 88(5A):95-145.

11. Stobberingh, E. E., H. J. van Eck, A. W. Houben, and C. P. A. van Boven. 1986. Analysis of the relationship between ampicillin resistance and $\beta$-lactamase production in Branhamella catarrhalis. Drugs 31:23-27.

12. Wallace, R. J., Jr., D. R. Nash, and V. A. Steingrube. 1990. Antibiotic susceptibilities and drug resistance in Moraxella (Branhamella) catarrhalis. Am. J. Med. 88(5A):465-505. 